\title{
Usefulness of the Combination of Tuberculin Skin Test and Interferon-Gamma Release Assay in Diagnosing Children with Tuberculosis
}

\author{
Hiroyuki Shimizu ${ }^{1,2}$ and Masaaki Mori ${ }^{3}$ \\ ${ }^{1}$ Children's Medical Center, Yokohama City University Medical Center, Yokohama, Kanagawa, Japan \\ ${ }^{2}$ Department of Infection Prevention and Control, Yokohama City University Medical Center, Yokohama, \\ Kanagawa, Japan \\ ${ }^{3}$ Department of Lifetime Clinical Immunology, Graduate School, Tokyo Medical and Dental University, Tokyo, \\ Japan
}

Japan is still designated as a medium incidence country, worldwide. Although the definitive diagnosis of tuberculosis is made by detecting pathogen from tissue specimens, collecting those from children is physically difficult. Therefore, we must frequently depend on the results of tuberculin skin test (TST) or interferon-gamma release assay (IGRA). However, the value of IGRA in diagnosing tuberculosis in infants remains unclear. We implemented TST and IGRA concurrently and examined the significance of combining these two tests by analyzing cases where conflicting results were evident from other tests. Subjects included 52 children ( 24 boys, 28 girls) age 15 years or younger ( $4.0 \pm 3.4$ years) who visited our hospital for close examination after medical checkup for tuberculosis contact tracing. The children underwent TST, $\mathrm{T}-S P O T^{\circledR} . T B$, chest plain radiography, and chest computed tomography with a view to tuberculosis diagnosis during a 26-months period from January 2013 to March 2015. T-SPOT results were positive in $9 / 52$ cases $(17.3 \%)$, and TST results were positive in $12 / 52$ cases $(23.1 \%)$. Conflicting results were observed in $7 / 52$ cases (13.5\%), and all cases were treated as latent tuberculosis infection or incident cases of tuberculosis. The value of T-SPOT in tuberculosis diagnosis in children is still being established. However, there are a number of cases where conflicting results for T-SPOT and TST are indicated. Thus, it is currently desirable to use T-SPOT and TST concomitantly for children at an early age, especially for children below the age of 5 years, as a diagnostic measure in complementing other techniques.

Keywords: Interferon-gamma release assay; latent tuberculosis infection; Mycobacterium tuberculosis; pediatric tuberculosis; tuberculin skin test

Tohoku J. Exp. Med., 2017 November, 243 (3), 205-210. C 2017 Tohoku University Medical Press

\section{Introduction}

The number of tuberculosis cases in children is decreasing in Japan with a similar decrease in adults. In recent years, fewer than 100 registered patients under 15 years old are diagnosed with tuberculosis annually (Tuberculosis Surveillance Center 2016). Accordingly, it is very rare for general pediatricians to see tuberculosis cases. However, the prevalence of tuberculosis among all age groups was 15.4 diagnosed cases for every 100,000 people in 2014 (Izumi et al. 2017); consequently, Japan is still designated as a medium incidence country, worldwide. Hence, tuberculosis cannot yet be considered a rare disease in
Japan. The source of infection in most instances of infant tuberculosis is adults, especially family members who have developed open tuberculosis. Therefore, it is important that latent tuberculosis infection (LTBI) cases be treated properly if the number of infant patients with tuberculosis is to remain small, given the prevalence of tuberculosis in Japan.

The definitive diagnosis of tuberculosis is made by detecting Mycobacterium tuberculosis from infected tissue specimens (Joos et al. 2006). However, collecting specimens from children is often physically difficult, and the positive rate in culture tests is also low when compared with adults (Cruz and Starke 2007). For those reasons, the tuberculin skin test (TST) has been used as a diagnostic

Received August 2, 2017; revised and accepted November 8, 2017. Published online November 28, 2017; doi: 10.1620/tjem.243.205.

Correspondence: Hiroyuki Shimizu, M.D. Ph.D., Department of Infection Prevention and Control, Yokohama City University Medical

Center, 4-57 Urafune-cho, Minami-ku, Yokohama, Kanagawa 232-0024, Japan.

e-mail: hiroyuki@yokohama-cu.ac.jp

Masaaki Mori, M.D. Ph.D., Department of Lifetime Clinical Immunology, Graduate School, Tokyo Medical and Dental University, 1-5-

45 Yushima, Bunkyo-ku, Tokyo 113-8510, Japan.

e-mail: mori.phv@tmd.ac.jp 
method for tuberculosis infection. TST is sensitive, but also reacts to Bacille de Calmette et Guerin (BCG). Therefore, the false-positive rate is a problem associated with TST. Consequently, the interferon-gamma release assay (IGRA) is often used as a technique to compensate for the shortcomings of TST. However, it has been suggested that the same sensitivity to IGRA in adults may not be present in infants without mature cell-mediated immunity (Jenum et al. 2014); namely, IGRA has excellent specificity but depends on the patient immune response.

Although some data suggest that IGRA is now useful for testing infants because IGRA technology has improved (Bianchi et al. 2009; Hesseling et al. 2009; Cruz et al. 2011; Kobashi et al. 2012), most of these studies have been conducted in patients who had already developed tuberculosis. Thus, it is unclear whether sensitivity is similar for cases with LTBI, where the patient still has a limited number of bacteria. In addition, it is inconclusive whether diagnosis of tuberculosis infection in children can be completely changed from use of the existing TST to the more recently developed IGRA.

To answer this clinical question, we implemented TST and IGRA for concurrent diagnosis of tuberculosis infection in infants and examined the significance of combining these two tests by analyzing cases where conflicting results were indicated using other tests.

\section{Materials and Methods}

\section{Study population}

Patients included 52 children age 15 years or younger who, at the request of a welfare and health center, visited Yokohama City University Medical Center for close examination after medical checkup for tuberculosis contact tracing. Patients underwent TST, T-SPOT $^{\circledR} . T B$ (T-SPOT; Oxford Immunotec Limited, Abingdon, United Kingdom), chest plain radiography and chest plain computed tomography (CT) with a view to tuberculosis diagnosis during a 26-month period from January 2013 to March 2015. We explained the significance and purpose of the simultaneous TST and T-SPOT testing, and obtained informed consent from all patients and their parents. Medical information, including the diagnostic result of TST, BCG vaccination history, findings from chest plain $\mathrm{CT}$, tuberculosis diagnosis (uninfected, LTBI, pulmonary tuberculosis) and treatments, was collected from a retrospective examination of electronic health records. This research has been approved by the ethics committee of Yokohama City University.

\section{Tuberculin skin test}

TST was performed using the Mantoux method by an experienced pediatrician. Injection of $10 \mathrm{U}$ of purified protein derivative was administered to the volar surface of each patient's forearm. The transverse redness and induration diameter was assessed 48 to 72 hours later by the ruler method. In Japan, routine BCG vaccination was started since 2005; therefore, most Japanese children are TST positive according to American Thoracic Society and Centers for Disease Control and Prevention recommendations. Thus, unique standards are in place to consider the potential for false-positive results caused by BCG vaccination in Japan. The criteria for TST determination used in this study were compliant with the standards of the Japanese Society for Tuberculosis (Table 1) (The Japanese Society for Tuberculosis 2006). A positive TST result was defined as an induration $>15 \mathrm{~mm}$ or redness $>30 \mathrm{~mm}$ according to those criteria.

\section{Interferon-gamma release assay (IGRA)}

Two IGRA methods, T-SPOT and QuantiFERON ${ }^{\circledR}$-TB Gold In-Tube assay (Cellestis Inc, Carnegie, Australia), are currently commercially available in Japan. We selected T-SPOT because this method has higher sensitivity in immunosuppressive conditions (Komiya et al. 2010; Cattamanchi et al. 2011) and requires less blood volume. At least $5 \mathrm{ml}$ of venous blood was collected into a heparinized blood-collecting tube which was performed along with blood cell count and biochemical examination, and the blood samples were outsourced for the assay. Peripheral blood mononuclear cells (PBMCs) were separated from the collected whole blood and adjusted to a prescribed cell number. Then, PBMC specimens were added to microplate wells with immobilized anti-IFN- $\gamma$ antibody, and reacted with two kinds of specific Mycobacterium tuberculosis antigens (ESAT-6 and CFP-10) for a period of between 16 and 20 hours. Wells were washed, and then labeled antibody reagent was added. The wells were washed again to remove unbound antibody, and then substrate was added. Finally, the number of T-cells producing IFN- $\gamma$ could be recognized as spots. The presence or absence of infection was determined based on the number of spots.

\section{Imaging test}

Front and lateral chest plain radiography as well as chest plain CT was implemented in all cases, as a previous series had suggested nodular shadows on chest CT images and evidence of germ discharge without obvious abnormal findings on chest plain radiographs (Ushiki et al. 2014). Therefore, chest CT was performed in all cases to maximize the sensitivity. Experienced radiologists read the images. Before each reading, the radiologist was informed about patients who were being examined for tuberculosis contact tracing.

Table 1. Criteria for significant reaction based on the results of the tuberculin test.

\begin{tabular}{|c|c|c|c|}
\hline & & \multicolumn{2}{|c|}{$\begin{array}{l}\text { history of exposure to } \\
\text { infectious pulmonary tuberculosis }\end{array}$} \\
\hline & & - & + \\
\hline \multirow{2}{*}{$\begin{array}{l}\text { history of BCG } \\
\text { vaccination }\end{array}$} & - & $\begin{array}{c}\text { induration } \geqq 15 \mathrm{~mm} \\
\text { or } \\
\text { redness } \geqq 30 \mathrm{~mm}\end{array}$ & $\begin{array}{c}\text { induration } \geqq 5 \mathrm{~mm} \\
\text { or } \\
\text { redness } \geqq 10 \mathrm{~mm}\end{array}$ \\
\hline & + & $\begin{array}{c}\text { induration } \geqq 20 \mathrm{~mm} \\
\text { or } \\
\text { redness } \geqq 40 \mathrm{~mm}\end{array}$ & $\begin{array}{c}\text { induration } \geqq 15 \mathrm{~mm} \\
\text { or } \\
\text { redness } \geqq 30 \mathrm{~mm}\end{array}$ \\
\hline
\end{tabular}




\section{Diagnosis}

There is no gold standard test for LTBI; therefore, we have no choice but to confirm positive T-SPOT or make a diagnosis based on the degree of exposure or duration of contact in a comprehensive way, regardless of the of T-SPOT or TST results. Diagnosis of pulmonary tuberculosis was made by a positive T-SPOT or TST result and abnormal findings on chest $\mathrm{CT}$ determined to not be any other respiratory infection. There were no pulmonary tuberculosis cases confirmed by culture or PCR.

\section{Results}

The subjects included 24 boys and 28 girls who were 15 years old or younger. The mean age was $4.0 \pm 3.4$ years. Five patients $(9.6 \%)$ were definitively diagnosed as uninfected for TST and T-SPOT negative with limited duration of contact with confirmed patient and low exposure risk, 40 patients $(76.9 \%)$ were diagnosed as LTBI, and seven $(13.5 \%)$ were diagnosed as incident cases of tuberculosis (Table 2). The details of LTBI cases are shown in Table 3. T-SPOT positive results comprised 9/52 patients (17.3\%), and TST positive results comprised $12 / 52$ patients $(23.1 \%)$. A total of $7 / 52$ patients $(13.5 \%)$ were positive for both T-SPOT and TST, and 38/52 patients (73.1\%) were negative for both T-SPOT and TST. There were 7/52 cases (13.5\%) with conflicting results. These included $2 / 52(3.8 \%)$ T-SPOT positive/TST negative cases, and 5/52 (9.6\%) T-SPOT negative/TST positive cases. The details of cases with conflicting results are shown in Table 4.

One T-SPOT positive/TST negative case was a 1-year old boy who had nodular shadows observed by CT. He was diagnosed with pulmonary tuberculosis and received multidrug therapy (2HRZ/4HR: 2 months of isoniazid, rifampicin and pyrazinamide and 4 months of isoniazid and rifampicin). Furthermore, one T-SPOT negative/TST positive case was an 11-month old girl who had nodular shadows observed by CT. She was diagnosed with pulmonary tuberculosis and received multidrug therapy (2HRZ/4HR). All other cases with conflicting results were diagnosed as having LTBI and were internally administered isoniazid for 6 months.

Of the 12 TST positive cases, the mean ages of seven T-SPOT positive cases and five T-SPOT negative cases were $8.0 \pm 9.4$ and $1.8 \pm 2.4$ years, respectively; indeed, the mean age of the latter group was significantly lower $(\mathrm{p}=$ $0.025)$.

\section{Discussion}

Making a definitive diagnosis of latent tuberculosis infection is not straightforward. When TST and T-SPOT assays were concurrently implemented for children who visited our hospital for a medical checkup for tuberculosis contact tracing, 7/52 cases (13.5\%) indicated conflicting results. If LTBI goes without treatment in younger children, there is a much higher possibility of severe tuberculosis developing (Marais et al. 2004). Therefore, when conflicting positive and negative results are obtained, it is better to adopt a precautionary stance and consider any conflicting result as a false-negative, thus providing the opportunity for proper treatment. This is preferable to considering a conflicting result as a false-positive.

There were two cases where a T-SPOT positive/TST negative pattern were observed in the present study. These results could be construed as false-positive by T-SPOT or false-negative by TST. For the former test, the T-SPOT is conducted with a reaction system using ESAT- 6 and CFP10 , which are human tuberculosis-specific antigens and are not affected by BCG (Lewinsohn 2009). Although some acid-fast bacilli, such as $M$. kansasii have been shown to cause T-SPOT positivity, a positive result by T-SPOT strongly suggests $M$. tuberculosis infection regardless of the TST result considering the pretest probability. For the latter instance, it may be that there was a technical error in the TST assay. Because TST is determined by intradermal inoculation, the result is a false-negative when the inoculation is done subcutaneously. In addition, the causes of false-negative results in TST include administration of corticosteroids and anergic conditions due to severe tuberculosis (Huebner et al. 1993). However, no immunosuppressive

Table 2. Number of tuberculosis diagnosis by the results of T-SPOT and TST.

\begin{tabular}{|c|c|c|c|c|}
\hline & & \multicolumn{2}{|c|}{ T-SPOT } & \multirow{2}{*}{ total } \\
\hline & & negative & positive & \\
\hline \multirow{4}{*}{ TST } & \multirow[b]{2}{*}{ negative } & $38(73.1 \%)$ & $2(3.8 \%)$ & $40(76.9 \%)$ \\
\hline & & $\begin{array}{l}5 \text { uninfected } \\
33 \text { LTBI }\end{array}$ & $\begin{array}{l}1 \text { LTBI } \\
1 \text { pulmonary tuberculosis }\end{array}$ & $\begin{array}{l}5 \text { uninfected } \\
34 \text { LTBI } \\
1 \text { pulmonary tuberculosis }\end{array}$ \\
\hline & \multirow[b]{2}{*}{ positive } & $5(9.6 \%)$ & $7(13.5 \%)$ & $12(23.1 \%)$ \\
\hline & & $\begin{array}{l}4 \text { LTBI } \\
1 \text { pulmonary tuberculosis }\end{array}$ & $\begin{array}{l}2 \text { LTBI } \\
5 \text { pulmonary tuberculosis }\end{array}$ & $\begin{array}{l}6 \text { LTBI } \\
6 \text { pulmonary tuberculosis }\end{array}$ \\
\hline \multirow{2}{*}{\multicolumn{2}{|c|}{ total }} & $43(82.7 \%)$ & $9(17.3 \%)$ & $52(100 \%)$ \\
\hline & & $\begin{array}{l}5 \text { uninfected } \\
37 \text { LTBI } \\
1 \text { pulmonary tuberculosis }\end{array}$ & $\begin{array}{l}3 \text { LTBI } \\
6 \text { pulmonary tuberculosis }\end{array}$ & $\begin{array}{l}5 \text { uninfected } \\
40 \text { LTBI } \\
7 \text { pulmonary tuberculosis }\end{array}$ \\
\hline
\end{tabular}

TST, tuberculin skin test; LTBI, latent tuberculosis infection. 
Table 3. Details of 40 LTBI cases which were diagnosed based on some circumstances.

\begin{tabular}{|c|c|c|c|c|c|c|c|c|c|c|c|c|}
\hline \multirow[b]{2}{*}{ Age } & \multirow[b]{2}{*}{ Sex } & \multicolumn{3}{|c|}{ T-SPOT } & \multicolumn{4}{|c|}{ TST (mm) } & \multirow[b]{2}{*}{ BCG } & \multirow{2}{*}{$\begin{array}{l}\text { Duration of } \\
\text { exposure } \\
\text { (hours) }\end{array}$} & \multirow{2}{*}{$\begin{array}{l}\text { Living together } \\
\text { with } \\
\text { confirmed patient }\end{array}$} & \multirow{2}{*}{$\begin{array}{c}\text { Other infected } \\
\text { patients among } \\
\text { screened population }\end{array}$} \\
\hline & & ESAT-6 & CFP-10 & & induration & redness & $\begin{array}{l}\text { double } \\
\text { redness }\end{array}$ & & & & & \\
\hline $2 y$ & $\mathrm{~F}$ & 0 & 0 & Negative & Unknown & $15 \times 5$ & - & Negative & Done & 30 & No & No \\
\hline $1 \mathrm{~m}$ & M & 0 & 0 & Negative & $0 \times 0$ & $0 \times 0$ & - & Negative & Not done & 9 & No & No \\
\hline $7 \mathrm{~m}$ & $\mathrm{~F}$ & 2 & 1 & Negative & $0 \times 0$ & $3 \times 3$ & - & Negative & Not done & 45 & No & No \\
\hline $5 y$ & M & 3 & 0 & Negative & $0 \times 0$ & $0 \times 0$ & - & Negative & Not done & 240 & No & No \\
\hline $8 \mathrm{~m}$ & M & 0 & 0 & Negative & $4 \times 4$ & $4 \times 4$ & - & Negative & Not done & Unknown & No & Yes \\
\hline $4 y$ & $\mathrm{~F}$ & -1 & -1 & Negative & $9 \times 6$ & $22 \times 18$ & - & Negative & Done & 42 & No & Yes \\
\hline $6 y$ & $\mathrm{~F}$ & 0 & 0 & Negative & $7 \times 7$ & $23 \times 18$ & - & Negative & Done & 192 & No & No \\
\hline $8 \mathrm{~m}$ & M & 0 & 0 & Negative & $0 \times 0$ & $2 \times 2$ & - & Negative & Done & 180 & No & No \\
\hline $3 y$ & $\mathrm{~F}$ & 0 & 0 & Negative & $5 \times 5$ & $13 \times 11$ & - & Negative & Done & 12 & No & No \\
\hline $3 y$ & F & 1 & 0 & Negative & $0 \times 0$ & $10 \times 6$ & - & Negative & Done & 68 & No & No \\
\hline $10 y$ & $\mathrm{~F}$ & 1 & 0 & Negative & $0 \times 0$ & $5 \times 4$ & - & Negative & Done & $>3$ months & Yes & Yes \\
\hline $8 y$ & M & 1 & 0 & Negative & $4 \times 3$ & $16 \times 12$ & - & Negative & Done & $>3$ months & Yes & Yes \\
\hline $4 y$ & M & 1 & 0 & Negative & $0 \times 0$ & $7 \times 6$ & - & Negative & Done & 13 & No & No \\
\hline $1 \mathrm{y}$ & M & 0 & 0 & Negative & $4 \times 3$ & $8 \times 6$ & - & Negative & Done & 13 & No & No \\
\hline $4 y$ & M & 1 & 0 & Negative & $3 \times 3$ & $11 \times 5$ & - & Negative & Done & $>3$ months & Yes & Yes \\
\hline $5 \mathrm{~m}$ & $\mathrm{~F}$ & 0 & 0 & Negative & $9 \times 8$ & $21 \times 16$ & - & Negative & Done & $>3$ months & Yes & Yes \\
\hline $1 y$ & $\mathrm{~F}$ & 0 & 0 & Negative & $8 \times 6$ & $20 \times 15$ & - & Negative & Done & 8 & No & No \\
\hline $2 y$ & M & 0 & 0 & Negative & $1 \times 1$ & $10 \times 9$ & - & Negative & Done & 8 & No & No \\
\hline $2 y$ & M & 1 & 0 & Negative & $4 \times 4$ & $9 \times 9$ & - & Negative & Done & 2months & Yes & No \\
\hline $7 y$ & $\mathrm{~F}$ & 0 & 0 & Negative & $0 \times 0$ & $16 \times 15$ & - & Negative & Done & $>3$ months & Yes & No \\
\hline $8 y$ & M & 0 & 0 & Negative & $7 \times 6$ & $16 \times 16$ & - & Negative & Done & $>3$ months & Yes & Yes \\
\hline $5 y$ & M & 0 & 0 & Negative & $0 \times 0$ & $6 \times 6$ & - & Negative & Done & $>3$ months & Yes & Yes \\
\hline $10 y$ & $\mathrm{~F}$ & 0 & 1 & Negative & $10 \times 9$ & $21 \times 16$ & - & Negative & Done & 24 & No & No \\
\hline $5 y$ & M & 0 & 0 & Negative & $6 \times 6$ & $8 \times 8$ & - & Negative & Done & 24 & No & No \\
\hline $1 y$ & $\mathrm{~F}$ & 2 & -2 & Negative & $9 \times 7$ & $22 \times 22$ & - & Negative & Done & $>3$ months & Yes & No \\
\hline $8 \mathrm{~m}$ & $\mathrm{~F}$ & 0 & 0 & Negative & $9 \times 7$ & $23 \times 22$ & - & Negative & Done & $>3$ months & Yes & No \\
\hline $3 y$ & F & 0 & 0 & Negative & $5 \times 5$ & $8 \times 8$ & - & Negative & Done & 24 & No & No \\
\hline $8 y$ & $\mathrm{~F}$ & 0 & 0 & Negative & $0 \times 0$ & $5 \times 4$ & - & Negative & Done & $>3$ months & Yes & Yes \\
\hline $4 y$ & M & 0 & 1 & Negative & $0 \times 0$ & $5 \times 4$ & - & Negative & Done & $>3$ months & Yes & Yes \\
\hline $3 y$ & M & 0 & 0 & Negative & $0 \times 0$ & $7 \times 6$ & - & Negative & Done & $>3$ months & Yes & No \\
\hline $4 y$ & $\mathrm{~F}$ & 0 & 0 & Negative & $0 \times 0$ & $4 \times 4$ & - & Negative & Done & 120 & Yes & No \\
\hline $4 y$ & M & 1 & 1 & Negative & $5 \times 4$ & $11 \times 9$ & - & Negative & Done & 72 & No & No \\
\hline $6 y$ & M & 0 & 0 & Negative & $4 \times 4$ & $9 \times 9$ & - & Negative & Done & 2 months & Yes & No \\
\hline $12 y$ & M & 43 & $>50$ & Positive & $11 \times 11$ & $11 \times 11$ & $36 \times 27$ & Positive & Done & $>3$ months & Yes & No \\
\hline $13 y$ & $\mathrm{~F}$ & 35 & $>50$ & Positive & $18 \times 15$ & $43 \times 32$ & - & Positive & Unknown & $>3$ months & Yes & Yes \\
\hline $5 y$ & F & 8 & 26 & Positive & $11 \times 11$ & $25 \times 16$ & - & Negative & Done & $>3$ months & Yes & Yes \\
\hline $4 y$ & F & 0 & 0 & Negative & $11 \times 11$ & $30 \times 21$ & - & Positive & Done & $>3$ months & Yes & No \\
\hline $1 y$ & F & 2 & 0 & Negative & $17 \times 15$ & $33 \times 22$ & - & Positive & Done & $>3$ months & Yes & No \\
\hline $2 y$ & $\mathrm{~F}$ & 0 & 0 & Negative & $7 \times 7$ & $7 \times 7$ & $21 \times 19$ & Positive & Done & 24 & No & No \\
\hline $1 \mathrm{y}$ & M & 1 & 0 & Negative & $17 \times 16$ & $18 \times 18$ & - & Positive & Done & 56 & No & No \\
\hline
\end{tabular}

LTBI, latent tuberculosis infection; TST, tuberculin skin test; BCG, Bacille de Calmette et Guerin.

agents were administered, and severe tuberculosis was not clinically suspected in the two cases in this study.

There were five cases where T-SPOT negative/TST positive was observed. This pattern may be caused by a false-negative result of the T-SPOT or a false-positive result of the TST. First, for the former suggestion, false-negative results of IGRA in young children who have no established cell-mediated immunity have been viewed with suspicion. A meta-analysis that examined the efficacy of T-SPOT in diagnosis of tuberculosis in children also pointed out the possibility that T-SPOT cannot accurately detect LTBI in children younger than 5 years old (Mandalakas et al. 2011). A possibility of false-negative results by T-SPOT due to young age was also fully assumed in our study because the mean age of the group of patients that were T-SPOT negative and TST positive was low. It is possible that a low specificity against acid-fast bacilli is possible if we consider the latter scenario. TST returns positive results against acid-fast bacilli, which include a number of non-tuberculous mycobacteria (Haimi-Cohen et al. 2001). In addition, cross-sensitivity with BCG is often a problem, especially in the Japanese population (Wang et al. 2002). BCG vaccination is regularly offered in Japan; therefore, the rate of BCG vaccination is very high in Japan. Accordingly, diagnosis of tuberculosis infection using TST should be made with caution. The Japanese Society for Tuberculosis has issued tentative standards that define strict criteria for determining positive cases as shown in Table 1. However, such stan- 
Table 4. Details of cases where T-SPOT and TST results conflicted.

\begin{tabular}{|c|c|c|c|c|c|c|c|c|c|c|c|c|c|}
\hline & \multirow{2}{*}{$\begin{array}{l}\text { Case } \\
\text { No. }\end{array}$} & \multirow{2}{*}{ Age } & \multirow{2}{*}{ Sex } & \multicolumn{2}{|c|}{ T-SPOT } & \multicolumn{3}{|c|}{ TST (mm) } & \multirow{2}{*}{ BCG } & \multirow{2}{*}{$\begin{array}{c}\mathrm{CT} \\
\text { findings }\end{array}$} & \multirow{2}{*}{ Culture } & \multirow{2}{*}{ Diagnosis } & \multirow{2}{*}{ Treatment } \\
\hline & & & & ESAT-6 & CFP-10 & induration & redness & double redness & & & & & \\
\hline \multirow{3}{*}{$\begin{array}{c}\text { T-SPOT positive } \\
\text { and } \\
\text { TST negative }\end{array}$} & 1 & $5 y$ & F & 8 & 26 & $11 \times 11$ & $25 \times 16$ & - & Done & n.p. & Not done & LTBI & $6 \mathrm{H}$ \\
\hline & 2 & $1 y$ & M & 36 & 2 & $7 \times 5$ & $13 \times 11$ & - & Done & nodule & Negative & Pulmonary TB & $2 \mathrm{HRZ}+4 \mathrm{HR}$ \\
\hline & 3 & $4 y$ & $\mathrm{~F}$ & 0 & 0 & $11 \times 11$ & $30 \times 21$ & - & Done & n.p. & Not done & LTBI & $6 \mathrm{H}$ \\
\hline \multirow{4}{*}{$\begin{array}{c}\text { T-SPOT negative } \\
\text { and } \\
\text { TST positive }\end{array}$} & 4 & $1 y$ & $\mathrm{~F}$ & 2 & 0 & $17 \times 15$ & $33 \times 22$ & - & Done & n.p. & Not done & LTBI & $6 \mathrm{H}$ \\
\hline & 5 & $2 y$ & F & 0 & 0 & $7 \times 7$ & $7 \times 7$ & $21 \times 19$ & Done & n.p. & Not done & LTBI & $6 \mathrm{H}$ \\
\hline & 6 & $1 \mathrm{y}$ & M & 1 & 0 & $17 \times 16$ & $18 \times 18$ & - & Done & n.p. & Negative & LTBI & $6 \mathrm{H}$ \\
\hline & 7 & $11 \mathrm{~m}$ & $\mathrm{~F}$ & 1 & 2 & $16 \times 14$ & $10 \times 6$ & $26 \times 24$ & Done & nodule & Negative & Pulmonary TB & $2 \mathrm{HRZ}+4 \mathrm{HR}$ \\
\hline
\end{tabular}

TST, tuberculin skin test; BCG, Bacille de Calmette et Guerin; CT, computed tomography; LTBI, latent tuberculosis infection.

dards cannot exclude the effect of BCG with absolute certainty. The reason for the low mean age of the group indicated as T-SPOT negative/TST positive might be because the reaction to TST was strong in young children due to the short period post-BCG vaccination.

As mentioned above, both false-positive and falsenegative results of TST cannot be ignored, and the sensitivity and specificity of these tests are not remotely acceptable as independent. However, TST is both inexpensive and easy to use, so its value is still evident in developing countries (Detjen et al. 2007; Tieu et al. 2014). The World Health Organization also recommends that priority be given to TST over IGRA, especially in countries with low-income and a high disease burden. There were $12 / 52$ cases (23.1\%) determined as being TST-positive in this study. Nodular shadows or swelling of the hilar area and mediastinal lymph nodes were observed by chest $\mathrm{CT}$ in six cases $(50 \%)$. Of the 40 TST-negative cases, however, similar findings by chest CT were observed only in four cases $(10 \%)$. These data are insufficient to fully discuss the sensitivity of TST for tuberculosis infection because no abnormal findings can be obtained by chest CT in LTBI, and there is no gold standard for diagnosis of LTBI. However, when the TST result was positive, abnormal findings were observed on CT in half the cases. In other words, considering the logistical ease of TST, its importance cannot be denied.

In conclusion, it is a responsible and inevitable decision to provide treatment for LTBI when at least one of these tests is positive because it is difficult to accurately identify the causes of conflicting results in TST and T-SPOT. Diagnosis of tuberculosis infection in children is difficult, and there are public health concerns that could significantly increase the risk of contracting the disease. Consequently, a certain degree of over-diagnosis cannot be avoided.

This study has several limitations. First, it was conducted in a single facility and with a small number of cases. Because infant tuberculosis has become rarer, a larger sample of cases from multiple facilities needs to be analyzed. Second, it is not possible to make a definitive diagnosis of LTBI. It is unclear whether the cases that indicated con- flicting results by T-SPOT and TST in this study were truly LTBI.

The value of T-SPOT in tuberculosis diagnosis in children is being established. However, there are a significant number of cases indicating conflicting results when both T-SPOT and TST are used. It is currently desirable to use T-SPOT and TST concomitantly for children at an early age, especially for those below the age of 5 years, as complementary diagnostic measures.

\section{Author Contributions}

Hiroyuki Shimizu: drafting the manuscript and figures. Masaaki Mori: conception and design of the study.

\section{Conflict of Interest}

Hiroyuki Shimizu declares no conflict of interest.

Masaaki Mori reports grants for Department of Lifetime Clinical Immunology from Chugai Pharmaceutical Co., Ltd., Ono Pharmaceutical Co., Ltd., Daiichi Sankyo Co., Ltd., Mitsubishi Tanabe Pharma Co., UCB Japan Co. Ltd., CSL Behring, Towa Pharmaceutical Co., Abbvie Japan Co., Ltd., Japan Blood Products Organization, Ayumi Pharmaceutical Co., and Nippon Kayaku Co., Ltd. Masaaki Mori has received lecture fees from MSD K.K and Abbvie LLC, and received consulting fees from Daiichi Sankyo Co., Ltd. and Taisho Pharmaceutical Co., Ltd.

\section{References}

Bianchi, L., Galli, L., Moriondo, M., Veneruso, G., Becciolini, L., Azzari, C., Chiappini, E. \& de Martino, M. (2009) Interferongamma release assay improves the diagnosis of tuberculosis in children. Pediatr. Infect. Dis. J., 28, 510-514.

Cattamanchi, A., Smith, R., Steingart, K.R., Metcalfe, J.Z., Date, A., Coleman, C., Marston, B.J., Huang, L., Hopewell, P.C. \& Pai, M. (2011) Interferon-gamma release assays for the diagnosis of latent tuberculosis infection in HIV-infected individuals: a systematic review and meta-analysis. J. Acquir. Immune Defic. Syndr., 56, 230-238.

Cruz, A.T., Geltemeyer, A.M., Starke, J.R., Flores, J.A., Graviss, E.A. \& Smith, K.C. (2011) Comparing the tuberculin skin test and T-SPOT.TB blood test in children. Pediatrics, 127, e31-38.

Cruz, A.T. \& Starke, J.R. (2007) Clinical manifestations of tuberculosis in children. Paediatr. Respir. Rev., 8, 107-117.

Detjen, A.K., Keil, T., Roll, S., Hauer, B., Mauch, H., Wahn, U. \& Magdorf, K. (2007) Interferon-gamma release assays improve 
the diagnosis of tuberculosis and nontuberculous mycobacterial disease in children in a country with a low incidence of tuberculosis. Clin. Infect. Dis., 45, 322-328.

Haimi-Cohen, Y., Zeharia, A., Mimouni, M., Soukhman, M. \& Amir, J. (2001) Skin indurations in response to tuberculin testing in patients with nontuberculous mycobacterial lymphadenitis. Clin. Infect. Dis., 33, 1786-1788.

Hesseling, A.C., Mandalakas, A.M., Kirchner, H.L., Chegou, N.N., Marais, B.J., Stanley, K., Zhu, X., Black, G., Beyers, N. \& Walzl, G. (2009) Highly discordant T cell responses in individuals with recent exposure to household tuberculosis. Thorax, 64, 840-846.

Huebner, R.E., Schein, M.F. \& Bass, J.B. Jr. (1993) The tuberculin skin test. Clin. Infect. Dis., 17, 968-975.

Izumi, K., Ohkado, A., Uchimura, K., Kawatsu, L., Suenaga, M., Urakawa, M. \& Kato, S. (2017) Evaluation of tuberculosis contact investigations in Japan. Int. J. Tuberc. Lung Dis., 21, 188-195.

Jenum, S., Selvam, S., Mahelai, D., Jesuraj, N., Cardenas, V., Kenneth, J., Hesseling, A.C., Doherty, T.M., Vaz, M. \& Grewal, H.M. (2014) Influence of age and nutritional status on the performance of the tuberculin skin test and QuantiFERON(R)-TB gold in-tube in young children evaluated for tuberculosis in Southern India. Pediatr. Infect. Dis. J., 33, e260-269.

Joos, L., Patuto, N., Chhajed, P.N. \& Tamm, M. (2006) Diagnostic yield of flexible bronchoscopy in current clinical practice. Swiss Med. Wkly., 136, 155-159.

Kobashi, Y., Abe, M., Mouri, K., Obase, Y., Miyashita, N. \& Oka, M. (2012) Usefulness of tuberculin skin test and three interferon-gamma release assays for the differential diagnosis of pulmonary tuberculosis. Intern. Med., 51, 1199-1205.

Komiya, K., Ariga, H., Nagai, H., Teramoto, S., Kurashima, A., Shoji, S. \& Nakajima, Y. (2010) Impact of peripheral lymphocyte count on the sensitivity of 2 IFN-gamma release assays, QFT-G and ELISPOT, in patients with pulmonary tubercu- losis. Intern. Med., 49, 1849-1855.

Lewinsohn, D.A. (2009) Embracing interferon-gamma release assays for diagnosis of latent tuberculosis infection. Pediatr. Infect. Dis. J., 28, 674-675.

Mandalakas, A.M., Detjen, A.K., Hesseling, A.C., Benedetti, A. \& Menzies, D. (2011) Interferon-gamma release assays and childhood tuberculosis: systematic review and meta-analysis. Int. J. Tuberc. Lung Dis., 15, 1018-1032.

Marais, B.J., Gie, R.P., Schaaf, H.S., Hesseling, A.C., Obihara, C.C., Starke, J.J., Enarson, D.A., Donald, P.R. \& Beyers, N. (2004) The natural history of childhood intra-thoracic tuberculosis: a critical review of literature from the pre-chemotherapy era. Int. J. Tuberc. Lung Dis., 8, 392-402.

The Japanese Society for Tuberculosis (2006) Tentative technological standard for tuberculin tests in the future. Kekkaku, 81, 387-391(in Japanese).

Tieu, H.V., Suntarattiwong, P., Puthanakit, T., Chotpitayasunondh, T., Chokephaibulkit, K., Sirivichayakul, S., Buranapraditkun, S., Rungrojrat, P., Chomchey, N., Tsiouris, S., Hammer, S., Nandi, V. \& Ananworanich, J.; Thai TB Px study group (2014) Comparing interferon-gamma release assays to tuberculin skin test in Thai children with tuberculosis exposure. PLoS One, 9, e105003.

Tuberculosis Surveillance Center (2016) Tuberculosis in Japanannual report 2016.

http://www.jata.or.jp/english/dl/pdf/TB_in_Japan_FINAL_ 1114.pdf [Accessed: November 6, 2017].

Ushiki, A., Yamazaki, Y., Ideura, G., Shinbo, T., Sugawara, M., Hama, M. \& Hanaoka, M. (2014) Pediatric pulmonary tuberculoma with a solid pulmonary nodule detected on chest computed tomography. Intern. Med., 53, 913-916.

Wang, L., Turner, M.O., Elwood, R.K., Schulzer, M. \& FitzGerald, J.M. (2002) A meta-analysis of the effect of Bacille Calmette Guerin vaccination on tuberculin skin test measurements. Thorax, 57, 804-809. 